\title{
PUTATIVE CUTANEOUS MANIFESTATIONS PERNIO LIKE AND COVID 19 INFECTION IN CHILDREN
}

\author{
Romina Gallizzi ${ }^{1}$, Diana Sutera ${ }^{2}$, Alessandra Spagnolo ${ }^{2}$, Serafinella Patrizia Cannavò ${ }^{3}$, \\ Loredana Grasso $^{4}$, Claudio Guarneri ${ }^{5}$, Giuseppe Nunnari ${ }^{6}$, Francesca Mazza ${ }^{2}$, and \\ Giovanni Pajno ${ }^{7}$ \\ ${ }^{1}$ University of Messina, Italy \\ ${ }^{2}$ Department of Human Pathology of Adulthood and Childhood Gaetano Barresi, Unit of \\ Pediatric, University of Messina, Italy \\ ${ }^{3}$ Department of Clinical and Experimental Medicine, Section of Dermatology, University of \\ Messina, Italy \\ ${ }^{4}$ Department of Services, Unit of Immunometry and Laboratory Diagnostic, University of \\ Messina, Italy \\ ${ }^{5}$ Department of Biomedical and Dental Sciences and Morphofunctional imaging, Institute \\ of Dermatology, University of Messina, Italy \\ ${ }^{6}$ Department of Clinical and Experimental Medicine, Unit of Infectious Diseases, \\ University of Messina, Italy \\ ${ }^{7}$ University of Messina
}

July 24, 2020

\begin{abstract}
ABSTRACT Background During the outbreak of COVID-19 many chilblain-like lesions are being increasingly reported. The aim of the study is to evaluate a possible correlation between pernio like skin lesions and SARS CoV2 infection. Methods All patients underwent not only clinical and laboratory tests to evaluate a possible underlying connective disease, but also specific SARS CoV2 investigations such as oropharyngeal swab and IgG-IgM serology. Results Nine patients, all children, were recruited. $6 / 9$ had respiratory and systemic symptoms that preceded cutaneous findings of approximately 2 weeks. The injuries observed were purplish, erythematous and edematous; four children reported subjective symptoms, painful and pruritus localized to the sole of the feet or to the toes, fingers or heels. Concerning blood exams: 3/9 had D-dimer weakly increased, 4 had ANA positivity: two with a title 1:160, one with 1:320 and one with 1:5120 and a speckled pattern. The latter patient had also ENA SS-A positive and FR positivity, confirmed at a second check, so as to allow us to make a diagnosis of connectivity. 4/9 had aPL positivity (IgM). Reactants acute phase were all negative. Oropharyngeal swabs and serology tests for SARS-CoV2 was negative (borderline in one patient for IgM). Conclusions Even if we do not have enough data to prove it, we hypothesize a correlation between chilblain and SARS-CoV2 infection for an increased number of these lesions described and also because such lesions appeared when temperatures were mild and patients were at home in isolation for the lockdown. Many questions remain open about interaction host-virus.
\end{abstract}

\section{PUTATIVE CUTANEOUS MANIFESTATIONS PERNIO LIKE AND COVID 19 INFEC- TION IN CHILDREN}

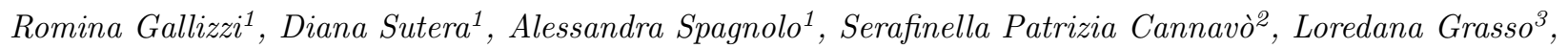
Claudio Guarneri4, Giuseppe Nunnari ${ }^{5}$, Francesca Mazza ${ }^{1}$, Giovanni Battista Pajno ${ }^{1}$ 
1 Department of Human Pathology of Adulthood and Childhood Gaetano Barresi, Unit of Pediatric, University of Messina, Italy

2 Department of Clinical and Experimental Medicine, Section of Dermatology, University of Messina, Italy

3 Department of Services, Unit of Immunometry and Laboratory Diagnostic, University of Messina, Italy, Messina, IT

4 Department of Biomedical and Dental Sciences and Morphofunctional imaging, Institute of Dermatology, University of Messina, Italy

5 Department of Clinical and Experimental Medicine, Unit of Infectious Diseases, University of Messina, Italy

Pernio like in children during Covid 19 era

*Corresponding Author Romina Gallizzi

Department of Human Pathology of Adulthood and Childhood Gaetano Barresi, University of Messina, Messina, Italy

Gaetano Martino University Hospital, UOC Pediatria

Via Consolare Valeria

Messina, 98125, Italy

Tel: 0902213154

Fax: 0902213170

E-mail: rgallizzi@unime.it

Romina Gallizzi : Conceptualization (Lead) Data curation (Lead) Investigation (Lead) Methodology (Lead) Project administration (Lead) Resources (Lead) Software (Lead) Supervision (Lead) Validation (Lead) Visualization (Lead) Writing-original draft (Lead) Writing-review \& editing (Lead)

Diana Sutera : Writing-original draft (Lead)

Alessandra Spagnolo : Writing-original draft (Lead)

Serafinella Patrizia Cannavò : Visualization (Equal)

Loredana Grasso : Methodology (Equal)

Claudio Guarneri : Visualization (Equal)

Giuseppe Nunnari : Visualization (Equal)

Francesca Mazza : Writing-original draft (Equal)

Giovanni Battista Pajno : Supervision (Equal) Validation (Equal) Visualization (Equal)

There aren't conflict of interests related to the manuscript content

We have not received financial support for the execution of the work

ABSTRACT

Background

During the outbreak of COVID-19 many chilblain-like lesions are being increasingly reported. The aim of the study is to evaluate a possible correlation between pernio like skin lesions and SARS CoV2 infection.

Methods 
All patients underwent not only clinical and laboratory tests to evaluate a possible underlying connective disease, but also specific SARS CoV2 investigations such as oropharyngeal swab and IgG-IgM serology.

Results

Nine patients, all children, were recruited. 6/9 had respiratory and systemic symptoms that preceded cutaneous findings of approximately 2 weeks. The injuries observed were purplish, erythematous and edematous; four children reported subjective symptoms, painful and pruritus localized to the sole of the feet or to the toes, fingers or heels. Concerning blood exams: 3/9 had D-dimer weakly increased, 4 had ANA positivity: two with a title 1:160, one with 1:320 and one with 1:5120 and a speckled pattern. The latter patient had also ENA SS-A positive and FR positivity, confirmed at a second check, so as to allow us to make a diagnosis of connectivity. 4/9 had aPL positivity (IgM). Reactants acute phase were all negative. Oropharyngeal swabs and serology tests for SARS-CoV2 was negative (borderline in one patient for IgM).

\section{Conclusions}

Even if we do not have enough data to prove it, we hypothesize a correlation between chilblain and SARSCoV2 infection for an increased number of these lesions described and also because such manifestations appeared when temperatures were mild and patients were at home in isolation for the lockdown. Many questions remain open about interaction host-virus.

KEYWORDS: chilblain, pernio, Covid19, children, skin lesions

The study was approved by our Institutional Ethical Committee and conducted in accordance with the Declaration of Helsinki.

\section{Introduction}

During the outbreak of COVID-19 many skin manifestations have been reported, and among these, in very significant numbers, newly eruptions like vascular and peculiar pernio-like skin lesions have been described in observational studies11Piccolo V, Neri I, Filippeschi C et Al. 2020 Chilblain-like lesions during COVID-19 epidemic: a preliminary study on 63 patients J Eur Acad Dermatol Venereol. 2020 Apr 24. doi: 10.1111/jdv.16526,22Galvan Casas C, Catala A, Carretero Hernandez G, et al. Classification of the cutaneous manifestations of COVID-19: a rapid prospective nationwide consensus study in Spain with 375 cases [published online ahead of print, 2020 Apr 29]. Br J Dermatol. 2020;10.1111/bjd.19163. doi:10.1111/bjd.19163,33Nerea Landa, Marta Mendieta-Eckert et al. Chilblainlike lesions on feet and hands during the COVID-19 Pandemic. International Journal of Dermatology. 2020 doi: 10.1111/ijd.14937'44Recalcati S, Barbagallo T, Frasin LA, et al. Acral cutaneous lesions in the time of COVID-19 [published online ahead of print, 2020 Apr 24]. J Eur Acad Dermatol Venereol . 2020;10.1111/jdv.16533. doi:10.1111/jdv.16533.Pernio, also referred to as chilblains, is a rare inflammatory condition. Chilblains derives from two Old English words "chill" (cold) and "blegen" (sore). Most commonly, pernio affects acral skin and develops among susceptible individuals who are exposed to cold, the lesions usually appear in fall or winter and disappear in spring or early summer. It is typically idiopathic and acute, nevertheless chronic forms also exist55Whitman PA, Crane JS, Pernio (Chilblains) StatPearls [Internet]. Treasure Island (FL): StatPearls Publishing; 2020-. 2019 Nov 4. The diagnosis of pernio is largely clinical and based on a thorough history and physical exam. The differential diagnosis must exclude diseases that can often be confused with other forms of pernio or vasculitic processes like LES or other conditions for example Raynaud phenomenon, acrocyanosis, cryoglobulinemia, cold panniculitis and Interferonopathies. The prognosis of pernio is good with minimal chronic sequelae.Single or multiple erythematous, purplish, edematous lesions appear, accompanied by intense pain, itching, or burning. Usually, pernio affects the toes and dorsum of the proximal phalanges66Tamara D. Simon, MD et Al. Pernio in Pediatrics.Pediatrics. 2005 Sep;116(3):e472-5.. Since March 2020, children with red and painful skin lesions, referable to chilblain have come to our attention. The injuries occurred in children and adolescents, however, in good health conditions, with suggestive history and established contact with SARS CoV2 patients. The increase in the number of cases of chilblain-like lesions compared to the cases with which we come into contact in our clinical practice 
every year, in association with the mild temperatures observed in those months in Southern Italy and the concomitant lockdown, led us to hypothesize a possible correlation with SARS Cov2 infection, so, the aim of the study is to evaluate this.

\section{Methods}

We evaluated the personal history and photographs of skin lesions of 26 patients, sent to us by their pediatrician, through multidisciplinary telematic meetings with dermatologists, rheumatological pediatricians, infectious disease specialist. We only included patients with pernio-like skin lesions (nine patients). We have excluded patients who were unable to perform the swab before hospital admission, patients whose parents did not obtain consent and patients with autoimmune diseases. We collected informed consent to obtain clinical information and photos of patients to be included in the study and to perform blood chemistry sampling. Therefore, we evaluated 9 cases of children who were referred to the Section of Pediatric Rheumatology, of General Pediatric Unit, department of Human Pathology in Adulthood and Childhood "G. Barresi", University of Messina, since March to April 2020 during outbreak of COVID-19. We have created a medical record in which we have included demographic information on patients, family and personal medical history, clinical manifestations and follow-up. We analyzed the photos of these patients at the time of the onset of symptoms and we assessed the cutaneous manifestations when they were admitted in the outpatient setting of the hospital. They underwent blood chemistry: a complete blood count (CBC), renal, hepatic, muscle function tests, urine test, complement levels, immunoglobulins, coagulation studies (prothrombin time, activated partial thromboplastin time, thrombin time and D-dimer test), rheumatoid factor (FR), antinuclear antibodies (ANA), extractable nuclear antigens (ENA), double stranded DNA (nDNA), anti-topoisomerase I (anti- SCL70), antiphospholipid antibodies (aPL), anti-cardiolipin (aCL), anti$\beta$-2-glycoprotein 1( $\beta 2 \mathrm{GP} 1$ ), and Lupus anticoagulant (LAC). In addiction, serology was performed using a chemiluminescent microparticle immunoassay (CLIA) for detection of IgG antibodies directed against the nucleocapsid protein of SARS-CoV2 and IgM.

\section{Results}

In our group of patients no significant difference in gender was detected ( 5 Females and 4 Males). The median age was 11,4 years (from 5 to 15 years). Two patients were siblings. All patients were from the South of Italy and live in the town of Messina and surroundings. Family history was negative for autoimmune disorders, Raynaud's phenomenon, acrocyanosis, chilblains or photosensitivity. All children were negative for clinical history of autoimmune or auto-inflammatory disorders except one with an episode of Raynaud's phenomenon a few years earlier. No family member of these patients presented symptoms attributable to SARS-CoV2 infection but their parents had contact with people for work reasons. Only two siblings had both parents with compatible symptoms and confirmed SARS-CoV2 infection: positive nasal swab. The cutaneous manifestations observed were purplish, erythematous and edematous, four children reported subjective symptoms, painful and pruritus localized to the sole of the feet or to the toes and/or fingers or heels (Fig. 1,2,3). Feet alone were mostly affected (6 of 9), hands (3 of 9) (Fig.1). Six patients had respiratory and systemic symptoms (cough, nasal congestion, chills, fever, asthenia) that preceded cutaneous findings of approximately 2 weeks (Table1). At the time of the first visit in 3 out 9 patients the skin lesions were quite mild only erythema persisted. Concerning the results of blood test: three out of nine had coagulation alterations (D-dimer weakly increased), 4 had ANA positivity: two with a title of 1:160, one with 1:320 and one with 1:5120 and a speckled pattern. The latter patient had also ENA SS-A positivity and FR positivity, in all others it was negative. Four out of nine had aPL positivity (IgM). Reactants acute phase were negative in all patients. Serology for SARS-CoV2 was negative (borderline in one patient for IgM). (Table2)

\section{Discussion}

During the SARS-Cov2 pandemic there have been numerous reports (case report, case series) in literatures of chilblain like lesions. In our clinical practice we come into contact with about 1-2 cases of chilblain/year, mainly in cold months. The increased demand for medical visits for these skin manifestations, associated to mild temperatures observed in those months in Southern Italy and the high diffusion of SARS Cov-2 
infection, led us to hypothesize a possible correlation with it.

We valued 9 cases of children who presented chilblain like lesions, since March to April 2020 during the outbreak of COVID-19. We performed first level tests, all negative except for D-dimer weakly increased in three of our patients. D-dimer is a marker of activation of coagulation and fibrinolysis and it provides a rapid evaluation of thrombotic activity. Its level correlated to coagulopathy have been described as prognostic factors in the evolution of SARS-CoV2 infection, especially in more severe patients. Zhang's study developed a triage, testing D-dimer levels at the admission, on the first and third day to predict survival in a cohort of patients and to evaluate management and follow up. The result was that a regulatory level of D-dimer at the presentation is highly predictive for survival11L, Yan X, Fan Q, Liu H, Liu X, Liu Z, Zhang Z. D-dimer levels on admission to predict in-hospital mortality in patients with Covid-19. J Thromb Haemost. 2020 Jun;18(6):1324-1329. doi: 10.1111/jth.14859. PMID: 32306492; PMCID: PMC7264730.. This is useful to highlight, as in our case, the D-dimer of our patients was weak is increased, a condition perfectly correlated with the mild symptoms of SARS-CoV2 putative infection presented. We have also done autoimmunity tests, three out of nine had a ANA positivity (speckled pattern): two with a title of 1:160, one 1:320 not confirmed at a subsequent check after two months; another one with 1:5120 (speckled pattern) and she also had FR positivity and ENA SS-A positivity. Four out of nine had aPL positivity (IgM). The findings of the high title positivity of the ANA, ENA and the FR, in patient $n$ 7, help us make a diagnosis of connectivity also for the similar symptomatology presented already in one occasion a year earlier. However, we cannot rule out that a possible SARS-CoV2 infection acted as a trigger. In other patients with ANA positivity, we can say that either it is a completely occasional finding, or more likely that this increase was caused by a recent viral infection (SARS-CoV2?). Acute viral, bacterial or parasitic infections may in fact induce antinuclear antibody and antiphospholipid antibodies positivity. This condition is transient and disappear within 2 or 3 months like in our patients22Berlin T, Zandman-Goddard G, Blank M, et al. Autoantibodies in nonautoimmune individuals during infections. Ann N Y Acad Sci . 2007;1108:584593. doi:10.1196/annals.1422.061. It is important to underline that the chilblain-like manifestations in our patients healed spontaneously without any treatment. The results of our study are homogeneous describing the negativity of the oropharyngeal swab and serological tests, carried out respectively after about 30 days and 60 days from the appearance of the systemic manifestations probably related to SARS-Cov2 infection (Table 1). A lack of seroconversion is hypothesized in asymptomatic or pauci-symptomatic subjects who had SARS-CoV2 infection. Zhang et al. showed that in a cohort of patients divided into three groups according to the severity of the symptoms, (severe, mild and asymptomatic): 100\% of the serious patients had shown seroconversion, only 1 out 5 asymptomatic cases generated specific antibody responses for SARSCoV-233Zhang Yongchen, Han Shen, Xinning Wang, Xudong Shi, Yang Li, Jiawei Yan, Yuxin Chen \& Bing $\mathrm{Gu}$ (2020) Different longitudinal patterns of nucleic acid and serology testing results based on disease severity of COVID-19 patients, Emerging Microbes \& Infections, 9:1, 833-836, DOI: 10.1080/22221751.2020.1756699. The case of our family is emblematic: two parents presented striking symptoms of SARS-Cov2 infection, very high fever and difficulty in breathing, and three positive swabs; the father had positive serology with very high IgG, such as to have allowed the donation of plasma for therapeutic purposes, and the mother instead, surprisingly,negative. Sons who came to our observation, both with chilblain, presented mild respiratory symptoms and they were negative for both swabs and IgG serology, while in one of the two cases, the IgM were borderline. Despite the negativities of diagnostic test for SARS-CoV2, we are still convinced that there is a correlation between this infection and the development of chilblain-like lesions, as described in numerous reports. A group of Italian Dermatologists described 63 patients with chilblain like lesions, primarily adolescents. Swab for Sars-Cov2 infection was performed in 11 patients and resulted positive in 2 cases; serology was available in 6 cases and it was positive in the 2 patients with positive swab ${ }^{2}$. Also a study among 375 cutaneous manifestations in suspected SARS-Cov2 patients in Spain, 71 (19\%) presented with "pseudo-chilblains". Only one of the 71 patients had previous history of chilblain; 29 (41\%) had SARS-CoV-2 confirmed $^{3}$. Recalcati et al described from Lombardy, 14 cases including 11 children (average age 14 years) and 3 young adults (average age 29 years) with chilblain-like eruptions. No systemic symptoms were reported, except a mild itch in 3 cases. Nasopharyngeal swab for SARS-CoV-2 gave negative results ${ }^{5}$. In a report of 19 adolescent patients with a clinical diagnosis of chilblain-like lesions nasopharyngeal swab and IgG serology for 
SARS-CoV-2 nucleocapsid protein were negative. Importantly, IgA serology for S1 domain of SARS-CoV-2 spike protein was positive in 6 patients and borderline in 3 patients44El Hachem M, Diociaiuti A, Concato C, et al. A clinical, histopathological and laboratory study of 19 consecutive Italian paediatric patients with chilblain-like lesions: lights and shadows on the relationship with COVID-19 infection [published online ahead of print, 2020 May 31]. J Eur Acad Dermatol Venereol. 2020;10.1111/jdv.16682. doi:10.1111/jdv.16682. The understanding of the immuno-pathogenetic mechanisms of interaction between the SARS-CoV2 infection and childrens is very intriguing but current knowledge does not seem to be sufficient. Why some of the children who come into contact with the SARS-CoV2 do not develop striking respiratory symptoms but present chilblain like lesions with negativity on diagnostic tests?

Matricardi et al developed the first model of interaction between the human immune system and SARS$\mathrm{CoV}-2$, as an attempt to produce a synthesis of what is known today55Matricardi PM, Dal Negro RW, Nisini R. The first, holistic immunological model of COVID-19: implications for prevention, diagnosis, and public health measures [published online ahead of print, 2020 May 2]. Pediatr Allergy Immunol. 2020;10.1111/pai.13271. doi:10.1111/pai.13271. What emerges is that innate immunity represents the first line of defense against the new SARS-CoV-2 and this first comparison establishes the natural history of the pathology, or if the infection will effectively block in the upper airways, or if the virus manages to reach the lungs. Innate immunity is essential for controlling virus replication before an adaptive immune response is generated66 Yu JC, Khodadadi H, Malik A, Davidson B, Salles ESL, Bhatia J, et al. Innate Immunity of Neonates and Infants. Front Immunol. 2018;9:1759. DOI:10.3389/fimmu.2018.01759.. Type I Interferones (IFN-I) are major components of the innate immune system and it represents critical antiviral molecules77Theofilopoulos AN, Baccala R, Beutler B, Kono DH. Type I interferons (a/b) in immunity and autoimmunity. Annu Rev Immunol 2005;23:307-35.. It is hypothesized that the IFN-I response may induce microangiopathic changes, producing chilblains and lupus-like erythematous eruption.

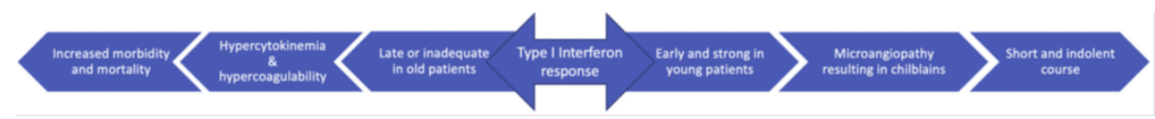

Coronavirus infection-induced chilblains11Kolivras A, Dehavay F, Delplace D, et al. Coronavirus (COVID19) infection-induced chilblains: A case report with histopathologic findings [published online ahead of print, 2020 Apr 18]. JAAD Case Rep. 2020;6(6):489-492.:

A mechanism has recently been considered to explain the appearance of autoimmune phenomena following the whole SARS-CoV2 infection: molecular mimicry22Cappello F Is. COVID-19 a proteiform disease inducing also molecular mimicry phenomena? Cell Stress Chaperones 2020 May;25(3):381-2. https://doi.org/10. 1007/s12192-020-01112-1.'33Sedaghat Z, Karimi N. Guillain Barre syndrome associated with COVID-19 infection: A case report. J Clin Neurosci 2020 Apr 15. https://doi.org/10.1016/j.jocn.2020. 04.062. pii: S0967-5868(20)30882-1.44Cappello F. COVID-19 and molecular mimicry: The Columbus' egg? J Clin Neurosci 2020 May 6. https://doi.org/10.1016/j.jocn.2020.05.015. pii: S0967-5868(20) 31156-5. Lucchesi and Floel55Lucchese G, Flöel A. Molecular mimicry between SARS-CoV-2 and respiratory pacemaker neurons. Autoimmun Rev 2020 May 1:102556https://doi.org/10.1016/j. autrev.2020.102556 have hypothesized a molecular mimicry mechanism between neuronal proteins present in the brain stem respiratory pacemaker neurons (DAB1, AIFM and SURF1) and viral epitopes of SARS-CoV2 treated antigenic. The same mechanism according to Angileri et al. could be responsible for anosmia, leukopenia and multi-organ failure caused by vascular damage, assuming they are associated with the molecular mimicry 66Angileri F. Molecular mimicry may explain multi-organ damage in COVID-19 Autoimmunity Reviews 2020 May 18 https://doi.org/10.1016/j.autrev.2020.102591.. A type 3 hypersensitivity therefore occurs with the deposition of antibody antigen complexes precipitate inside the tissues, in particular the blood vessels, inducing a serious inflammatory state by the action of the complement anaphylatoxins (C3a and C5a), which in turn stimulate the release of histamine from mast cells and the recruitment of phagocytes, first in neutrophils, the main cause of tissue damage and following «leukocytoclastic vasculitis» (LCV), also reported in the English 
medical literature from the term «hypersensitivity vasculitis77C.A. Janeway Jr., P. Travers, M. Walport, M. Shlomchik, Immunobiology: the immune system in health and disease, fifth ed., Garland Science, New York, 2001,88D. Baigrie, P. Bansal, A. Goyal, J.S. Crane, Leukocytoclastic vasculitis (hypersensitivity vasculitis), StatPearls Publishing, Treasure Island, 2020». This pathogenic mechanisms could explain the appearance of chilblain-like lesions due to SARS COV-2 infection. In conclusion we think there is a correlation between chilblain like lesion and SARS-Cov2 infection, but further studies are needed to prove it. We hypothesize this for an increased number of this skin lesions described in the literature during this period, as in our experience, and also because such lesions appeared at a time when temperatures were mild in Southern Italy and patients were at home because of the lockdown. To our knowledge, this is one of the few studies that collects a series of patients with chilblain like lesions, evaluating the possible association with COVID 19 (oropharyngeal swab and serology test) but also for rheumatological diseases. Many questions remain open about interaction host-virus.

ACKNOWLEDGMENTS : Thanks to Italian Federation of Pediatrician (FIMP) of Messina for collaboration.

\section{IMPACT STATEMENT}

During the outbreak of COVID-19 many chilblain-like lesions are being increasingly reported.The increase in the number of cases of chilblain-like lesions compared to the cases with which we come into contact in our clinical practice every year, in association with the mild temperatures observed in those months in Southern Italy and the concomitant lockdown, led us to hypothesize a possible correlation with SARS Cov 2 infection. To our knowledge, this is one of the few studies that collects a series of patients with chilblain like lesions, evaluating the possible association with COVID 19 (oropharyngeal swab and serology test) but also for rheumatological diseases.

\section{REFERENCES}

\section{Hosted file}

tabelle da caricare.docx available at https://authorea.com/users/328512/articles/471898putative-cutaneous-manifestations-pernio-like-and-covid-19-infection-in-children

\section{Hosted file}

foto pernio1.docx available at https://authorea.com/users/328512/articles/471898-putativecutaneous-manifestations-pernio-like-and-covid-19-infection-in-children

\section{Hosted file}

foto pernio 2.docx available at https://authorea.com/users/328512/articles/471898-putativecutaneous-manifestations-pernio-like-and-covid-19-infection-in-children

\section{Hosted file}

foto pernio 3.docx available at https://authorea.com/users/328512/articles/471898-putativecutaneous-manifestations-pernio-like-and-covid-19-infection-in-children 